\title{
Preliminary antimicrobial susceptibility: possibility of early and effective therapy during treatment of septic patients
}

\author{
Maria Antonietta Distasi, Giuseppe Mennea', Angela Pirronti ${ }^{2}$,Tito Del Gaudio \\ U.O.C. di Patologia Clinica P.O. di Andria ASL BT \\ I U.O.C. di Medicina Interna P.O. di Andria ASL BT \\ 2 U.OC. di Anestesia e Rianimazione P.O. di Andria ASL BT
}

Key words: Direct antimicrobial susceptibility, E-test, Blood culture, MBQ

Antibiogramma preliminare: possibilità di una terapia precoce ed efficace nel paziente settico

\section{SUMMARY}

Blood cultures have the drawback of requiring long time to be carried out. Our study was designed with the aim of early reports on both causing agents and preliminary antimicrobial susceptibility. We carried out a direct antimicrobial susceptibility on 79 positive blood cultures (42 for Gram-negative bacteria of which 2 I MDRO; 22 for staphylococci of which $15 \mathrm{~S}$. aureus; and 15 Enterococci/Streptococci) employing E-test strips deposited on Müller-Hinton Agar for staphylococci and Gram-negative bacteria, and Müller-Hinton Agar $+5 \%$ of blood for enterococci/streptococci. MICs were compared to the antimicrobial susceptibility tests carried out using Vitek2. On the basis of the interpretative criteria (SIR), the comparison between the direct antimicrobial susceptibility and the routine antimicrobial susceptibility, showed a concordance from $92.6 \%$ up to $100 \%$ for Gram-negative bacteria; from $90.0 \%$ up to $100 \%$ for staphylococci; from $86.7 \%$ up to $100 \%$ for streptococci/enterococci, respectively. With regard to MICs comparison, good concordance was detected on the basis of \pm 1 doubling dilution. Moreover, this work allowed, in the case of Gram-negative bacteria, either the early identification of multidrug-resistant organisms or providing the MIC evaluation in a broader interval than it would be possible by the reference antimicrobial susceptibility.

\section{INTRODUZIONE}

I tempi di refertazione del campione positivo rappresentano una delle criticità dell'attività del Laboratorio di Microbiologia. In particolare, nel caso dell'emocoltura, il risultato definitivo può richiedere un tempo anche maggiore delle previste 48 ore dall'accettazione del campione. L'emocoltura costituisce l'esame più richiesto sia per l'individuazione dell'agente eziologico della sepsi che per l'inizio della terapia antibiotica mirata. La prognosi quoad vitam del paziente settico è tanto più favorevole quanto più è breve il tempo che intercorre fra l'accertamento della positività dell'emocoltura e l'inizio della terapia antibiotica. Dati della letteratura confermano una concreta riduzione della mortalità in relazione alla precocità ed all'adeguatezza dell'intervento terapeutico $(1,2)$. Pertanto abbiamo valutato una metodologia di intervento sul flacone di emocoltura positivo che ci consentisse di offrire preliminarmente in 18/24 ore sia un'indicazione del microrganismo responsabile che l'antibiogramma $(\mathrm{ABG})$, in termini di indice di sensibilità (SIR) e di Minima Concentrazione Inibente (MIC). A questo scopo sono state utilizzate le strisce di E-test.
La selezione degli antibiotici è stata effettuata in base alla scelta integrata fra la nostra proposta laboratoristica e l'esperienza dei clinici responsabili del trattamento del paziente settico. L'ultima fase del lavoro è stata poi quella di mettere a confronto i risultati ottenuti con la suddetta metodica preliminare con il nostro metodo di riferimento.

\section{MATERIALI E METODI}

Nel biennio 2011-2012, presso 1'U.O. di Patologia Clinica del Presidio Ospedaliero di Andria, sono state eseguite 5.168 emocolture su 1.433 pazienti; $326(22.7 \%)$ presentavano batteriemia e tra questi 79 sono stati selezionati per il nostro studio. Il campione positivo è stato seminato sulla batteria di terreni prevista dalla nostra procedura di routine. Dopo incubazione di $24 \mathrm{~h}$ a $37^{\circ} \mathrm{C}$, le colonie sviluppate sono state avviate all'identificazione biochimica ed all'antibiogramma utilizzando il sistema Vitek2 (bioMérieux, Italia), nostro sistema di riferimento $(3,4)$. Contestualmente alla semina del campione sono stati allestiti 2 strisci di sangue, colorati rispettivamente con il metodo di Gram e con blu di metilene. In caso di osservazione di cocchi Gram positivi, per individuare 
$S$. aureus abbiamo effettuato il test di coagulasi diretta, diluendo $50 \mu 1$ di brodocoltura in $0.5 \mathrm{ml}$ di plasma citratato ed osservando l'eventuale formazione di coagulo dopo incubazione a $37^{\circ} \mathrm{C}$ per $4 \mathrm{~h}$ (5). Dopo l'esame batterioscopico per la definizione microscopica del microrganismo, è stato allestito l'antibiogramma diretto. È stata preparata una sospensione diluendo 5 gocce di brodocoltura in $5 \mathrm{ml}$ di soluzione fisiologica con successiva semina su terreno Müller-Hinton Agar per stafilococchi e bacilli Gram negativi e su terreno Müller-Hinton Agar $+5 \%$ di sangue di montone per enterococchi/streptococchi (6).

$\mathrm{Su}$ questi terreni sono state applicate le strisce di E-test di:

a) colistina, piperacillina/tazobactam, meropenem, ciprofloxacina per i bacilli Gram negativi;

b) teicoplanina, linezolid, levofloxacina, tobramicina per stafilococchi;

c) vancomicina, linezolid, imipenem, ampicillina/sulbactam per enterococchi/streptococchi (Figura I).

Le MIC osservate sono state confrontate con quelle dell'antibiogramma di riferimento: per l'interpretazione della sensibilità sono stati utilizzati i criteri CLSI fino al marzo 2011 e, successivamente, quelli EUCAST.

\section{RISULTATI}

Le caratteristiche morfologiche e tintoriali dei 79 microrganismi considerati sono state confermate dall'identificazione ottenuta con il nostro sistema di riferimento (42 bacilli Gram negativi dei quali 21 MDR; 22 stafilococchi di cui 15 S. aureus e 15 enterococchi/streptococchi) (Tabella 1). Dal confronto tra l'antibiogramma diretto e quello definitivo, considerando i criteri interpretativi (SIR) abbiamo osservato una concordanza compresa tra $92.6 \%$ e $100 \%$ per bacilli Gram negativi; tra $90.9 \%$ e $100 \%$ per stafilococchi; tra $86.7 \%$ e $100 \%$ per enterococchi/streptococchi. Il confronto tra le MIC dell'ABG diretto e quello di riferimento è stato effettuato considerando una differenza di \pm 1 diluizione e \pm 2 diluizioni della scala graduata dell'E-test. Nel primo caso abbiamo osservato una concordanza compresa tra $81.5 \%$ e $95.1 \%$ per bacilli Gram negativi; tra $47.6 \%$ e $86.4 \%$ per stafilococchi; tra $33.3 \%$ e $100 \%$ per enterococchi/streptococchi; nel secondo caso la concordanza è risultata tra $85.2 \%$ e $97.6 \%$ per bacilli Gram negativi; tra $66.7 \%$ e $95.8 \%$ per stafilococchi; tra $66.7 \%$ e $100 \%$ per enterococchi/streptococchi (Tabella 2).

\section{DISCUSSIONE}

L'analisi dei nostri risultati ci ha permesso di osservare una concordanza ottimale fra identifica- zione preliminare e identificazione definitiva. Quanto al confronto dei risultati relativi agli agenti antimicrobici, la concordanza è risultata ottimale fra gli indici SIR. Per quanto riguarda il confronto fra le MIC, per poter affermare la validità dei risultati ottenuti nell'indagine preliminare, abbiamo fatto riferimento alle linee guida (7) secondo le quali il metodo in valutazione, rispetto al metodo di riferimento, deve dare risultati uguali o entro \pm 1 diluizione a raddoppio. Abbiamo potuto verificare buona coincidenza delle MIC ottenute con i due metodi quando il valore è stato confrontato a \pm 2 diluizioni della scala graduata dell'E-test corrispondente ad 1 diluizione a raddoppio. Tuttavia è stata evidenziata la bassa percentuale di concordanza delle MIC per Linezolid testato per stafilococchi, così come tutte le percentuali di concordanza dell'Imipenem testato per enterococchi/streptococchi sono risultate le più basse della nostra osservazione. Nel caso del Linezolid il valore di MIC è stato costantemente sottostimato, laddove abbiamo notato una sovrastima del valore della MIC dell'Imipenem. Inoltre il Linezolid deposto su Müller-Hinton Agar ha presentato, dopo $24 \mathrm{~h}$ di incubazione, scarsa definizione dell'alone di inibizione con conseguente imprecisione nella definizione della MIC. Invece gli ottimi dati di concordanza del Linezolid testato per enterococchi/streptococchi su terreno Müller-Hinton Agar $+5 \%$ di sangue di montone ha permesso di verificare su questo terreno, anche in meno di $24 \mathrm{~h}$, una definizione più netta dell'alone di inibizione e, di conseguenza, anche una valutazione più precisa della MIC. Il metodo di lavoro è stato pertanto modificato, successivamente all'elaborazione dei risultati, utilizzando anche per l'ABG preliminare per stafilococchi il terreno Müller-Hinton Agar $+5 \%$ di sangue di montone. Di particolare interesse ci è sembrata inoltre l'osservazione che con l'E-test fosse possibile fornire un valore di MIC in un range di diluizioni più ampio rispetto al range del nostro metodo di riferimento (Figura II). Questo è un dato di notevole rilievo sia per l'importanza della MIC ai fini di un impiego mirato del farmaco che per la possibilità di determinare il rapporto fra la MIC e i breakpoint del farmaco stesso. Il quoziente MICbreakpoint (MBQ) è quello che definisce più concretamente le proprietà farmacodinamiche dell'antimicrobico e rappresenta uno strumento più utile per una scelta terapeutica mirata (8). È evidente che questo è meno significativo per valori di MIC inferiori a quelli più bassi ottenibili con il metodo di riferimento, mentre acquista un rilievo terapeutico per tutte le MIC definite al di sopra della diluizione più alta prevista dal metodo standard. Una definizione più precisa del valore della 
MIC nell'ambito di una resistenza dell'antimicrobico consente un uso più accurato del valore della MIC stessa ed un calcolo preciso del MBQ. Questo risulterebbe utile per consentire di sfruttare in modo ottimale l'effetto dose dipendente del farmaco, per valutare l'ipotesi di una terapia di associazione e per tentare un sinergismo farmacologico. Con il nostro lavoro è stato possibile valutando gli E-test alle 18-24h individuare e segnalare precocemente i microrganismi MDR ed offrire la possibilità di una scelta terapeutica. La segnalazione di MIC di resistenza numericamente definite ha potuto favorire una gestione più adeguata di dose, modo e tempo di somministrazione di uno o più farmaci e l'eventuale de-escalation di quanto considerabile, alla luce dei risultati, inadeguato. Con questo metodo di lavoro abbiamo attuato un tentativo di migliorare il flusso operativo su campioni critici come le emocolture positive riducendo in maniera significativa il Turnaround Time (TAT) di refertazione (9). Questo miglioramento tecnico si è tradotto in un supporto di rilievo per il clinico sulla tempestività e sull'adeguatezza del trattamento antimicrobico.

Infine, dobbiamo considerare che un metodo operativo più costoso a causa dell'uso dell'E-test si traduce in una ipotesi di risparmio sia per la spesa farmacologica, data la possibilità di mirare la scelta terapeutica, sia per i tempi di gestione e degenza del paziente.

Tabella I. Microrganismi testati con antibiogramma diretto

\begin{tabular}{lcllll}
\hline \multicolumn{2}{l}{ BACILLI GRAM NEGATIVI } & \multicolumn{2}{l}{ STAFILOCOCCHI } & \multicolumn{2}{l}{ ENTEROCOCCHI/STREPTOCOCCHI } \\
\hline E. coli & $N^{\circ}$ & & $N^{\circ}$ & & $N^{\circ}$ \\
\hline A. baumannii & 12 & S. aureus & 10 & E. faecium & E. faecalis \\
\hline K. pneumoniae MDR & 9 & MRSA & 5 & S. pneumoniae & \\
\hline K. pneumoniae & 7 & S. hominis & 3 & \\
\hline P. aeruginosa & 3 & S. epidermidis & 3 & \\
\hline P. mirabilis & 5 & S. haemolyticus & I & & \\
\hline S. marcescens & 4 & & & & \\
\hline E. cloacae & $\mathrm{I}$ & & & TOTALE & 15
\end{tabular}

Tabella 2. Percentuali di concordanza tra antibiogramma diretto ed antibiogramma di riferimento

BACILLI GRAM NEGATIVI

COLISTINA PIPERACI

TAZOBACTAM

MEROPENEM CIPROFLOXACINA

\begin{tabular}{lllll}
\hline SIR & $100 \%$ & $92.6 \%$ & $92.6 \%$ & $100 \%$ \\
\hline MIC \pm I DIL & $88.1 \%$ & $81.5 \%$ & $88.1 \%$ & $95.1 \%$ \\
\hline MIC \pm 2 DIL & $90.5 \%$ & $85.2 \%$ & $88.1 \%$ & $97.6 \%$ \\
\hline
\end{tabular}

STAFILOCOCCHI

\begin{tabular}{lcccc}
\hline & TEICOPLANINA & LINEZOLID & LEVOFLOXACINA & TOBRAMICINA \\
\hline SIR & $95.5 \%$ & $100 \%$ & $90.9 \%$ & $95.5 \%$ \\
\hline MIC \pm I DIL & $72.7 \%$ & $47.6 \%$ & $72.7 \%$ & $86.4 \%$ \\
\hline MIC \pm 2 DIL & $1.8 \%$ & $66.7 \%$ & $86.4 \%$ & $95.8 \%$ \\
\hline
\end{tabular}

ENTEROCOCCHI/STREPTOCOCCHI

\begin{tabular}{lcccc}
\hline & VANCOMICINA & LINEZOLID & IMIPENEM & $\begin{array}{c}\text { AMPICILLINA } \\
\text { SULBACTAM }\end{array}$ \\
\hline SIR & $100 \%$ & $100 \%$ & $86.7 \%$ & $100 \%$ \\
\hline MIC \pm I DIL & $33.3 \%$ & $86.7 \%$ & $53.3 \%$ & $100 \%$ \\
\hline$M I C \pm 2$ DIL & $100 \%$ & $93.3 \%$ & $66.7 \%$ & $100 \%$ \\
\hline
\end{tabular}




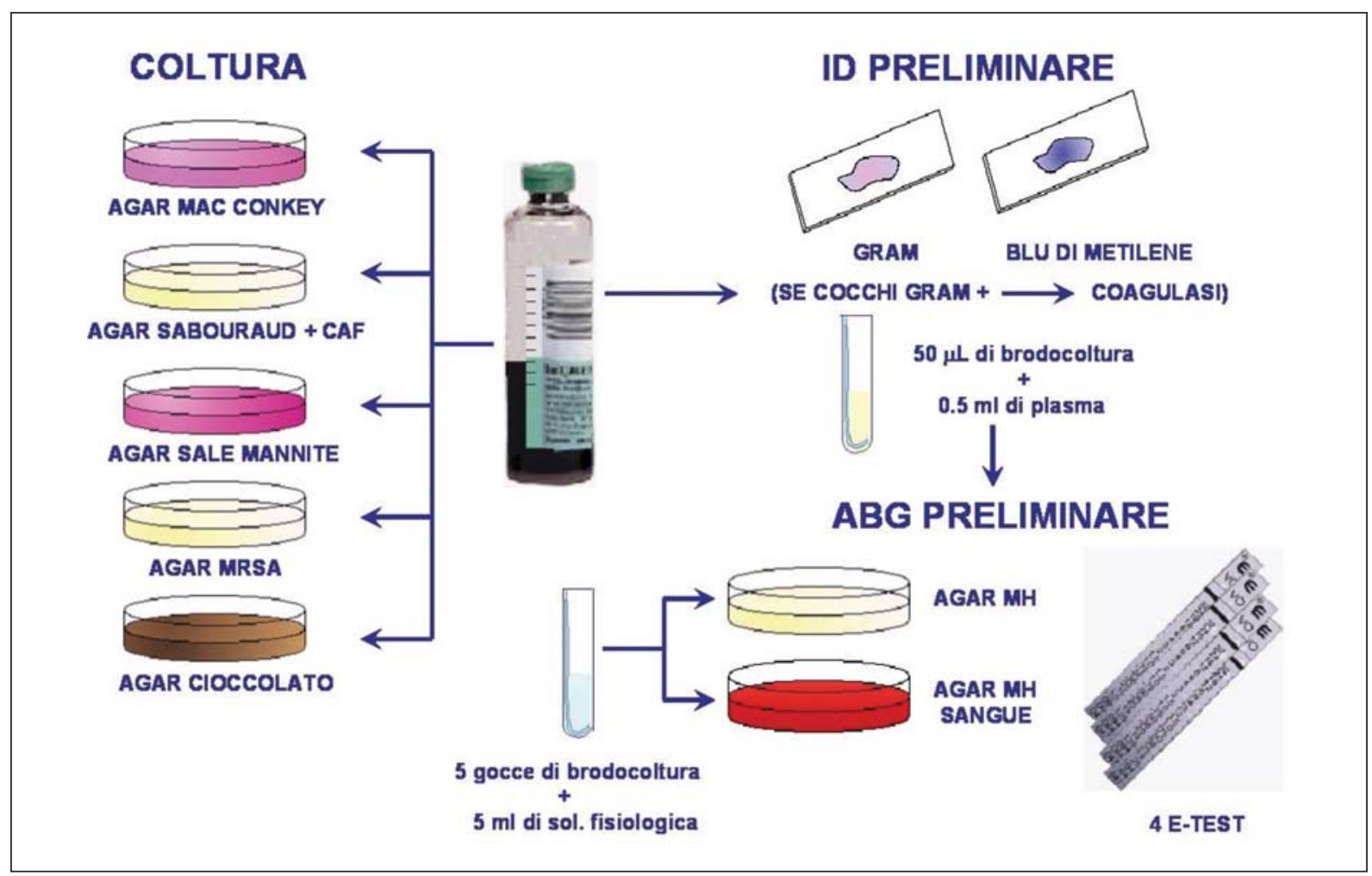

Figura I. Gestione dell'emocoltura positiva

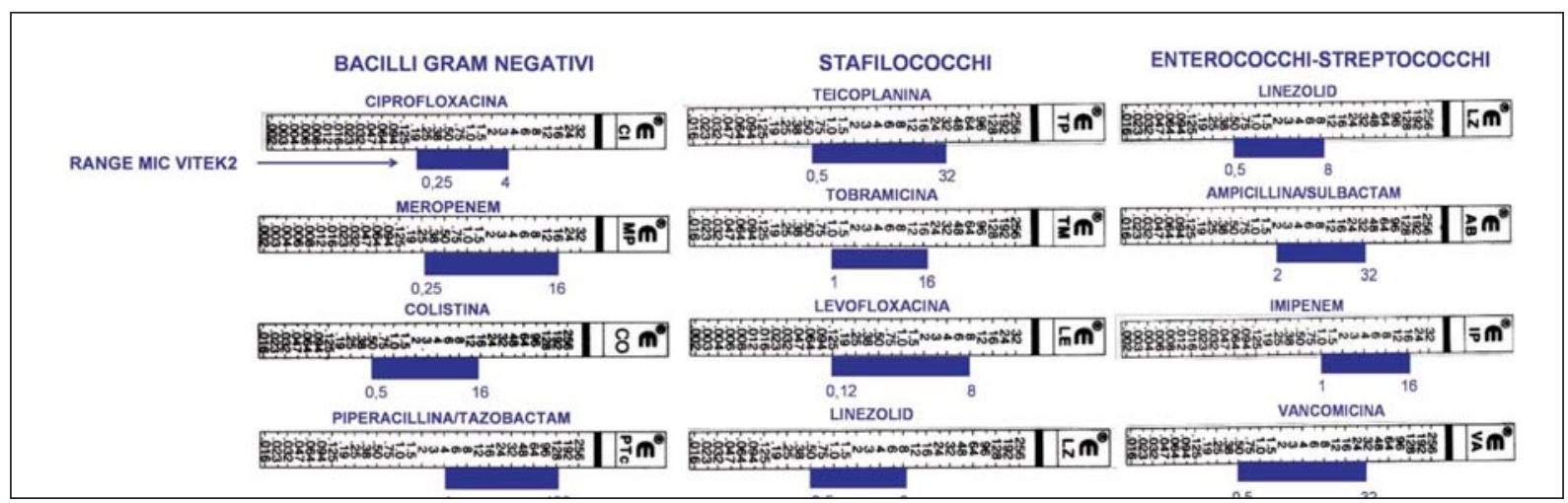

Figura II. Differenza tra il range della MIC dell'E-test e il range della MIC del sistema VITEK2

\section{BIBLIOGRAFIA}

1. Kroumova V, Gobbato E, Macaluso P, et al. Preliminary indications for antibiotic susceptibility tests in less than six hour in positive blood cultures. Microb Med. 2010; 25(1): 24-6.

2. Ledonne R, Cavalcanti P, Filia MA, et al. Sepsis: a more faster report from blood culture. Microb Med. 2010; 25(3): 193-4.

3. Soloaga RN, Carrion NA, Margari AM, et al. Evaluation of direct susceptibility testing from blood culture bottles. Clinical usefulness. Rev Argent Microbiol. 2012; 44(3): 165-9.,

4. Romero-Gómez MP, Gómez-Gil R, Paño-Pardo JR, Mingorance J. Identification and susceptibility testing of microorganism by direct inoculation from positive blood culture bottles by combining MALDI-TOF and Vitek-2 Compact is rapid and effective. $J$ Infect. Dec 2012; 65(6): 513-20.
5. Elizabeth D, Hermsen ED, Shull SS, et al. Pharmacoeconomic Analysis of Microbiologic Techniques for Differentiating Staphylococci Directly from Blood Culture Bottles. J Clin Microbiol. 2008; 46: 2924-9.

6. Edelmann A, Tim Pietzcker $\mathrm{T}$, Wellinghausen N. Comparison of direct disk diffusion and standard microtitre broth dilution susceptibility testing of blood culture isolates. J Med Microbiol. 2007; 56: 202-7.

7. Guidance for Industry and FDA. Class II Special Controls Guidance Document: Antimicrobial Susceptibility Test (AST) Systems. 2009; 36.

8. Scaglione F, Thomson RB, Mohr JF, Howe R. MIC $\mathrm{PK} / \mathrm{PD}$ per un approccio razionale alla terapia antibiotica. Documenta. 2005; 15: 13-5.

9. Grosso S, Camporese A. Valutazione di appropriatezza, efficienza ed efficacia di alcune procedure analitiche per ridurre il turnaround time delle emocolture. IJLAM 2007; 3: 203-11. 